\title{
Extended-Release Formulation and Medication Adherence
}

John A. Romley, $P h D^{1,2}$, Zhiwen Xie, $M A^{3}$, Tommy Chiou, BA ${ }^{3}$, Dana Goldman, $P h D^{1,2}$, and Anne L. Peters, $M D^{4}$

${ }^{1}$ Price School of Public Policy, University of Southern California, Los Angeles, CA, USA; ${ }^{2}$ USC School of Pharmacy, Los Angeles, CA, USA; ${ }^{3}$ USC Schaeffer Center for Health Policy \& Economics, Los Angeles, CA, USA; ${ }^{4}$ Keck School of Medicine, Los Angeles, CA, USA.

$\mathrm{J}$ Gen Intern Med 35(1):354-6

DOI: $10.1007 / \mathrm{s} 11606-019-05275-1$

(C) Society of General Internal Medicine 2019

\section{INTRODUCTION}

Suboptimal adherence to chronic medications undermines treatment effectiveness and costs the USA as much as \$289 billion annually. ${ }^{1}$ Prescription of extended-release (XR) formulations may offer a partial solution, as past studies have found that XR formulations can improve adherence. ${ }^{2}$ However, the majority of past studies have analyzed adherence to XR formulations only in the context of specific medications and short-term time frames ( $<1$ year). ${ }^{3,4}$ This study investigates the effects of XR on long-term ( $\geq 1$ year) adherence across 15 chronic medications, as well as the influence of cost-sharing on XR usage.

\section{METHODS}

An IRB exempted this study. We identified patients treated with chronic medications from a $25 \%$ sample of Optum's Clinformatics® claims from 2011 to 2013. Patients were between 18 and 64, had at least two fills of $\mathrm{XR} /$ non-XR formulations of a selected medication, initiated treatment at least 1 year before December 31, 2013, and remained continuously enrolled in the same insurance plan until December 31, 2013. Adherence to a given drug/ formulation was measured at the patient level via variable medication possession ratio (MPR), defined as the sum of days' supply (regardless of dosage) from first to last prescription (inclusive) divided by the time between the last and first prescription dates plus last days' supply, without truncation. ${ }^{5}$ Patients who took both formulations were excluded.

In total, 15 medications were analyzed (Fig. 1). Selected medications had both XR and non-XR formulations as of
December 31, 2010, were intended for long-term use and had at least 100 users of each formulation.

To examine the effects of XR formulations, we performed linear regressions to determine adjusted MPR, controlling for age, race, income, education, geographic area of residence, the Charlson comorbidity index, number of drugs taken concurrently, and plan type. We also defined adherence as MPR > 0.85 and generated adjusted adherence rates using logistic regression with the same controls.

We also studied patients who initiated treatment in 2011 and calculated two fixed MPRs (denominator =365), one beginning from a patient's first fill to 1 year after treatment initiation, and another for the second year after initiation, with excess days' supply truncated. ${ }^{5}$

Finally, we calculated out-of-pocket costs and conducted linear regressions to analyze the relationship between proportion of XR users and difference in out-of-pocket cost between $\mathrm{XR}$ and non-XR across the medications.

Average adjusted MPRs, adherence rates, and out-ofpocket costs were weighted by total days' supply of medications. Analyses were conducted using Stata (version 14.1).

\section{RESULTS}

A total of 123,243 patients (55\% XR users) were included in our sample, with mean follow-up of 845 days, average time on medication of 631 days, and median of 12 fills. XR users showed significantly higher adjusted MPR $(p<0.01)$ than non-XR users in 13 of the 15 drugs analyzed (Fig. 1). Average adjusted MPRs were greater for XR users $(80.2 \%)$ than for non-XR users (74.8\%) (difference, $5.4 \%$ (95\% CI, 4.1$6.8 \%)$ ). Average adjusted adherence was $56.3 \%$ among XR users and $46.0 \%$ among non-XR users (difference, $10.3 \%$ (95\% CI, 7.4-13.3\%)).

Among patients with 2 years of follow-up, average adjusted MPRs were greater among XR users during both year 1 


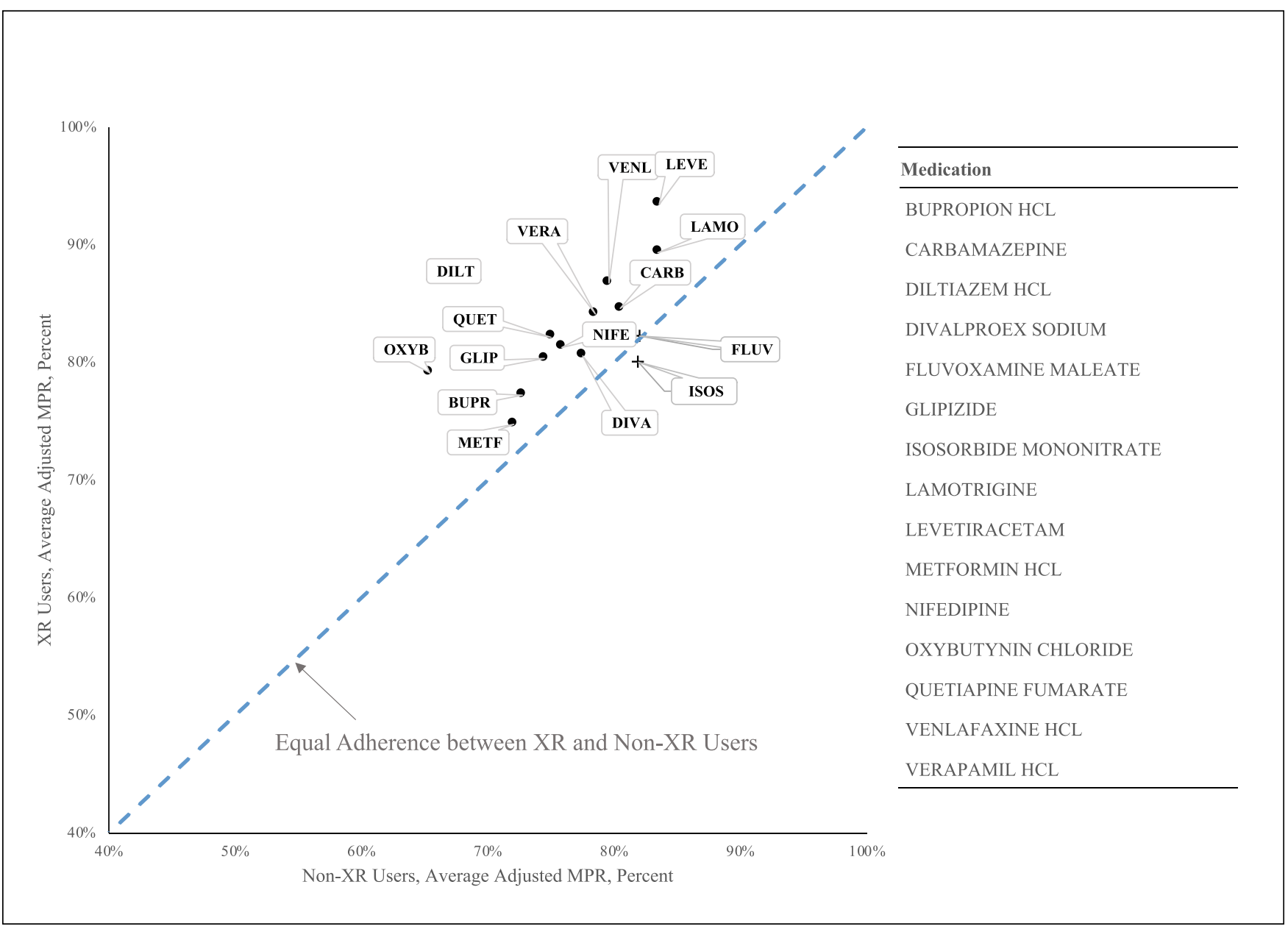

Figure 1 The relationship between extended-release (XR) formulation and medication possession ratio. XR, extended release; MPR, medication possession ratio. Dots indicate drugs for which difference between XR and non-XR MPR is statistically significant $(p<0.05)$, while plus signs indicate insignificant differences. MPR was measured as the sum of days' supply from first to last prescription (inclusive) divided by the time between the last and first prescription dates plus last days' supply, without truncation. Adjusted MPR adherence rates were calculated from multivariate regression models, with patient-level MPR as the dependent variable. The models controlled for age, race, income, education, geographic area of residence, the Charlson comorbidity index, number of drugs taken concurrently, and plan type.

(75.6\% vs $68.0 \%$; difference, $7.6 \% ; 95 \%$ CI $5.1-11.3 \%)$ and year 2 (57.8\% vs $49.6 \%$; difference, $8.2 \%$; $95 \%$ CI 5.1 $11.3 \%)$.

Average out-of-pocket costs for a 30-day supply were higher for XR medications (difference, \$7.46). The proportion of XR users and relative cost of XR were negatively correlated (correlation coefficient, - 0.79) (Fig. 2). A one standard deviation (\$19.63) decrease in additional cost of XR formulations (around its mean) was associated with an increase of 36.0 percentage points $(95 \% \mathrm{CI}, 5.8-66.2 \%, p=0.023)$ in the proportion of XR users.

\section{DISCUSSION}

Average medication adherence was higher among patients treated with XR formulations, to a degree that is clinically significant. For instance, a 5.4\% improvement in adherence to preventive medications after myocardial infarction is associated with an $11 \%$ reduction in rate of major vascular events or revascularization. ${ }^{6}$

XR formulations had higher costs, which were associated with lower proportion of XR users. Prior literature has found a negative relationship between copayments and medication adherence. ${ }^{1}$ More generous coverage of XR formulations could therefore be an effective solution for patients treated with chronic medications.

Limitations of the study include a selective patient population, discrepancies between observed and actual adherence, and unexamined reasons for patients switching between formulations. 


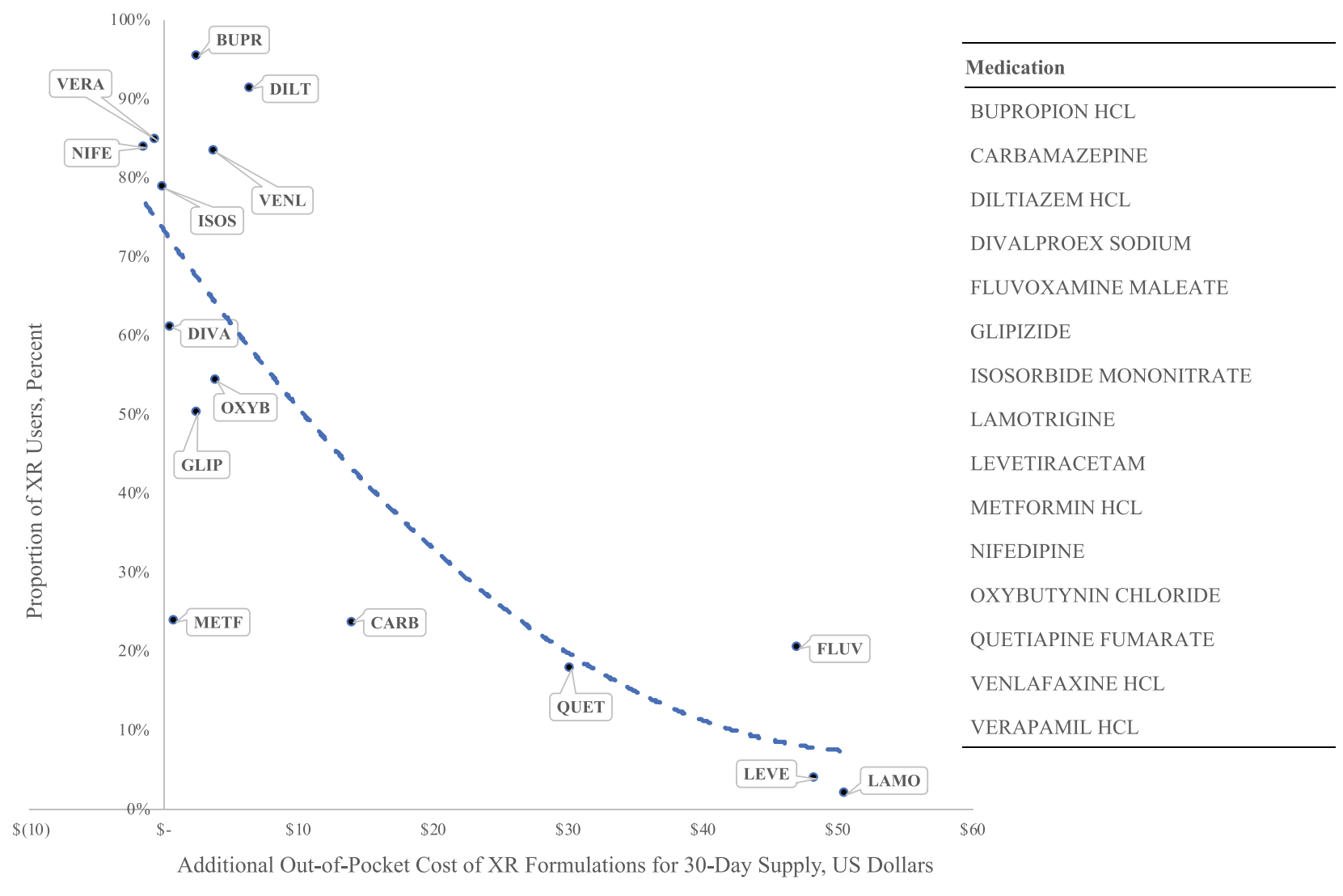

Figure 2 The relationship between additional out-of-pocket cost of extended-release (XR) formulations and proportion of XR users. XR, extended release. Out-of-pocket cost per day of supply was calculated as the sum of deductible and copay, divided by total days of supply. The chart presented the out-of-pocket cost for 30-day supply. Additional out-of-pocket cost for XR formulations was calculated as the difference in out-of-pocket cost between XR and non-XR formulations. Positive additional cost meant XR formulations had higher out-of-pocket costs. All costs were measured in US dollars. The fitted line is based on a linear regression model that adjusted for heterogeneity. The equation for the fitted line is: Proportion of $X R=0.734-0.0249 \times$ Additional Cost of $X R+0.0002 \times$ Additional Cost of $X R^{2}$. Key model statistics: $N=15, R^{2}=$ $0.637, p$ value $<0.01$ for the regression.

\section{Contributors: None.}

Corresponding Author: John A. Romley, PhD; USC School of Pharmacy, Los Angeles, CA, USA (e-mail: romley@healthpolicy.usc. edu).

Funders Research reported in this publication was supported by the National Institute on Aging of the National Institutes of Health under Award Number 5P30AG024968.

\section{Compliance with Ethical Standards:}

Conflict of Interest: The authors declare that they do not have a conflict of interest.

\section{REFERENCES}

1. Viswanathan $\mathbf{M}$, Golin $\mathbf{C E}$, Jones $\mathbf{C D}$, et al. Interventions to improve adherence to self-administered medications for chronic diseases in the united states: a systematic review. Ann Intern Med. 2012;157(11):785795.

2. Ingersoll KS, Cohen J. The impact of medication regimen factors on adherence to chronic treatment: a review of literature. J Behav Med. 2008;31(3):213-224.

3. Wang $\mathbf{L}$, Sun $\mathbf{X}, \mathbf{D u} \mathbf{L}$, et al. Effects and patient compliance of sustainedrelease versus immediate-release glipizides in patients with type 2 diabetes mellitus: a systematic review and meta-analysis. J Evid Based Med. 2011;4(4):232-241.

4. Adler LD, Nierenberg AA. Review of Medication Adherence in Children and Adults with ADHD. Postgrad Med. 2010;122(1):184191.

5. Sperber CM, Samarasinghe SR, Lomax GP. An upper and lower bound of the medication possession ratio. Patient Prefer Adherence. $2017 ; 11: 1469$

6. Choudhry NK, Avorn J, Glynn RJ, et al. Full coverage for preventive medications after myocardial infarction. N Engl J Med. 2011;365(22):20882097.

Publisher's Note Springer Nature remains neutral with regard to jurisdictional claims in published maps and institutional affiliations. 